\section{MAGE-A 3/4 and NY-ESO-1 antigens expression in metastatic esophageal squamous cell carcinoma}

\author{
T. Bujas, ${ }^{1}$ Z. Marušić, ${ }^{2}$ M. Perić Balja, ${ }^{2}$ \\ A. Mijić, ${ }^{3}$ B. Krušlin,, ${ }^{2,4}$ D. Tomas ${ }^{2,4}$ \\ 'Department of Pathology, General \\ Hospital Karlovac; ' 2 Department \\ of Pathology, Sestre milosrdnice \\ University Hospital, Zagreb; \\ ${ }^{3}$ Department of Surgery, Sestre \\ milosrdnice University Hospital, Zagreb; \\ ${ }^{4}$ School of Medicine, University \\ of Zagreb, Croatia
}

\section{Abstract}

In the present study we analyzed immunohistochemical expression of MAGE-A 3/4 and NY-ESO-1 in 55 samples of esophageal squamous cell carcinomas (ESCC) and their respective lymph node metastases. To our knowledge this is the first study to assess and compare the expression of these antigens in ESCC lymph node metastases.

Fifty $(90.9 \%)$ primary ESCC were positive for MAGE-A 3/4 and 53 (96.6\%) were positive for NY-ESO-1. MAGE-A 3/4 was expressed in all lymph node metastases and the intensity of expression was high in a majority of cases. NYESO-1 was negative in 2 (7.1\%) lymph nodes metastases, while the reaction was predominantly moderate in the positive group. In primary tumors MAGE-A 3/4 showed a significantly higher intensity of expression compared to NY-ES0-1 ( $\mathrm{P}=0.047)$, while in lymph node metastases the intensity of expression was not significantly different $(\mathrm{P}=0.387)$. Primary tumors with and without lymph node metastases showed no significant differences in MAGE-A $3 / 4 \quad(\mathrm{P}=0.672)$ and NY-ESO-1 $(\mathrm{P}=0.444)$ expression. Intensity of MAGE-A $3 / 4$ $(\mathrm{P}=0.461)$ and NY-ESO-1 $(\mathrm{P}=0.414)$ expression in primary tumors was not significantly different compared to the expression in their respective lymph nodes metastases. Expression of MAGE-A 3/4 in primary tumors showed significant positive correlation with primary tumor expression of NY-ESO-1 $(\mathrm{P}=0.021)$ but no significant correlation with the expression of MAGE-A 3/4 in lymph node metastases $(\mathrm{P}=0.056)$. Expression of NY-ESO1 in primary tumors showed significant positive correlation with the expression of NYESO-1 in lymph node metastases $(\mathrm{P}=0.001)$ and significant negative correlation with patients' age $(\mathrm{P}<0.001)$. Expression of MAGEA 3/4 and NY-ESO-1 in primary tumors and lymph node metastases showed no significant correlation with prognostic parameters such as tumor grade and TNM stage $(\mathrm{P}>0.05)$. We have shown different levels of MAGE-A $3 / 4$ and NY-ESO-1 expression in almost all specimens of primary tumor and lymph node metastases, suggesting that ESCC may be possible target of immunotherapy and anti-tumor vaccination. High levels of expression in lymph node metastases indicate possible clinical benefit of postoperative vaccine with MAGE-A3 and NY-ESO1 in advanced stage of disease.

\section{Introduction}

Esophageal squamous cell carcinoma (ESCC) accounts for up to 6\% of all gastrointestinal cancers. It has a highly unfavorable prognosis with a five-year survival rate of less than $10 \%$. Due to current lack of early screening strategies, ESCC often presents in an advanced stage at the time of diagnosis. As a result, its mortality rate is almost equal to its incidence (5 per 100,000 population). ${ }^{1}$ Current treatment has not provided much hope for a positive outcome, and a great deal of research is under way with the scope of detecting new therapeutic methods.

High risk of metastatic recurrence suggests that cell dissemination may occur early in the course of ESCC; thus, it seems that active immunotherapy may deserve a place among treatment modalities. $^{2}$

Tumor associated antigens (TAA) are specific antigens that may be recognized by cytotoxic $\mathrm{T}$ lymphocytes when presented within the major histocompatibility complex (MHC) class I molecules. Among tumor antigens, cancer/ testis antigens (CTA) such as MAGE and NY-ESO-1 have received particular attention as potential targets for vaccine-based immunotherapy of cancer because they are not expressed in normal human tissues, with the exception of male germline cells and placenta, and are activated in a number of tumors. ${ }^{3,4}$

The MAGE genes are the best characterized members of the CTA family. Currently, there are 23 related MAGE genes divided into four clusters on chromosome X. ${ }^{5}$ More recently, monoclonal antibodies to certain MAGE antigens have been developed, allowing for their immunohistochemical localization within neoplastic tissues and the pattern of antigen expression was described in a subset of uterine malignancies, ${ }^{6}$ skin melanoma,${ }^{7}$ lung, ${ }^{8}$ rena $^{9}$ and esophageal carcinomas. ${ }^{10-14}$

NY-ESO-1 is another member of the CTA family that was originally isolated from esophageal carcinoma. It is one of the most immunogenic CTA known to date. NY-ESO-1 elicits a strong, integrated humoral and cellu-
Correspondence: Dr. Davor Tomas, Department of Pathology, Sestre milosrdnice University Hospital, Vinogradska 29, 10000 Zagreb, Croatia. Tel. +385.1.3787465 - Fax: +385.1.3787244. E-mail: dtomas@kbsm.hr

Key words: esophageal squamous cell carcinoma, metastases, MAGE-A 3/4, NY-ESO-1.

Contributions: TB, histopathological evaluation, manuscript writing and revision; ZM, MPB, literature search, photomicrographs acquisition, material preparation; AM, patient clinical information supply, manuscript drafting; BK, participation in the histopathological evaluation, manuscript drafting; DT, participation in the histopathological evaluation, outlined manuscript general concept outlining, manuscript drafting and revision. All authors have read and approved the final manuscript.

Conflict of interest: the authors declare no potential conflict of interest.

Received for publication: 14 January 2011. Accepted for publication: 15 February 2011.

This work is licensed under a Creative Commons Attribution 3.0 License (by-nc 3.0).

(C) Copyright T. Bujas et al., 2011

Licensee PAGEPress, Italy

European Journal of Histochemistry 2011; 55:e7 doi:10.4081/ejh.2011.e7

lar immune response in a high proportion of patients with NY-ESO-1-expressing tumors. ${ }^{15}$ Expression of NY-ESO-1 in normal tissue is restricted to the testis. Its expression has been described in a number of malignancies including lung, ${ }^{8}$ renal, ${ }^{9}$ prostate, ${ }^{16}$ penile squamous ${ }^{17}$ and esophageal carcinomas. ${ }^{14,18-20}$

Recent studies have outlined MAGE and NYESO-1 expression as a potential target in immunotherapy and anti-tumor vaccination..$^{14,21-24}$

In this study we analyzed MAGE-A $3 / 4$ and NY-ESO-1 immunohistochemical expression in ESCC samples and their lymph node metastases, in order to determine the connection between individual antigen expression in primary and metastatic lesions. In addition, the expression of mentioned antigens was correlated with some clinico-pathological tumor characteristics.

\section{Materials and Methods}

\section{Patients}

Fifty-five patients with ESCC who underwent radical surgery at the University 
Department of Surgery, Sestre milosrdnice University Hospital, Zagreb, between January 1, 2003 and December 31, 2007 were included in the study. None of the patients received preoperative chemotherapy or radiotherapy. There were 8 (14.5\%) female and 47 (85.5\%) male patients, aged 38 to 73 years (mean 57.2 years). Tumor diameter varied from 1.4 to 10 $\mathrm{cm}$ (mean $3.6 \mathrm{~cm}$ ). Eighteen (32.7\%) tumors were located in the upper, $32(58.2 \%)$ in the middle and $5(9.1 \%)$ in the lower part of the esophagus. The pathologic stage of each tumor at the time of operation was defined according to the TNM system..$^{25}$ There were 4 (7.3\%) T1, 20 (36.3\%) T2, 28 (50.9\%) T3 and 3 (5.5\%) T4 tumors. Twenty eight (50.9\%) patients had one or more lymph node metastases. All dissected lymph nodes were completely processed for pathological examination. The number of dissected lymph nodes varied from 3 to 24 (mean 10.5 nodes). Three to 22 lymph nodes (mean 9.8 nodes) were dissected in cases with negative lymph nodes, and 4 to 24 lymph nodes (mean 11.1 nodes) in cases with positive lymph nodes. In cases with positive lymph nodes, 1 to 12 nodes (mean 3.2 nodes) were affected by tumor. Each tumor also received a histological grade based on parameters of mitotic activity, anisonucleosis and degree of differentiation, as described in the World Health Organization classification. ${ }^{1}$ Seven (12.7\%) tumors were grade $1,32(58.2 \%)$ grade 2 and $16(29.1 \%)$ grade 3.

\section{Methods}

Tumors were fixed in $10 \%$ buffered formalin for approximately $24 \mathrm{~h}$, cut at 3-4 milimeters and sampled in 3-7 sections. The specimens were embedded in paraffin, routinely cut and stained with hematoxylin and eosin (H\&E). In each case, the available H\&E sections were reviewed and slides with the deepest portion of tumor penetration were selected for immunohistochemical analysis.

Two monoclonal antibodies were used to determine the expression of analyzed proteins in primary tumors and lymph node metastases (both antibodies are gift from Dr. Spagnoli, Basel, Switzerland). 57B was generated on immunization of mice with recombinant MAGE-A3 ${ }^{26}$ However, this antibody recognizes a variety of MAGE-A molecules, and it is considered a multi-MAGE-A-specific reagent, but a recent analysis indicates it to be mainly reactive to MAGE-A3 and MAGE-A4..$^{27,28}$ D8.38 antibody, recognizing NY-ESO-1 and its homologous LAGE-1 CTA, has been previously described ${ }^{29}$ Tissue sections of 3-5 $\mu \mathrm{m}$ thickness were cut from paraffin embedded tissue blocks, placed on object slides (Menzel-Glaser, Braunschweig, Germany), and incubated for $20 \mathrm{~min}$ in a thermostat at $60^{\circ} \mathrm{C}$. The sections were then deparaffinized and incubated for
$3 \times 5 \mathrm{~min}$ in $10 \mathrm{mmol} / \mathrm{L}$ of citrate buffer $(\mathrm{pH} 6.0)$ in a microwave oven at $800 \mathrm{~W}$. Subsequently, tissue slides were washed with phosphate buffered saline (PBS) buffer (pH 7.2), and endogenous peroxidase activity was blocked by a 5-min treatment with hydrogen peroxide (No. S2023, Dako, Carpinteria, CA, USA). Slides were then washed with PBS-buffer and incubated for 90 min with MAGE-A 3/4 57B or NY-ESO-1 D8.38 undiluted supernatants at room temperature. After washing in PBS, slides were incubated with a secondary biotinylated antibody (No. K0690, Dako) for 30 min. Slides were then washed with PBS-buffer and treated with streptavidin-horseradish peroxidase (No. K0690, Dako) for $30 \mathrm{~min}$. Tissue sections were washed once more in PBSbuffer, and then Chromogen (No. K3468, Dako) was added for 5 min. Slides were washed in distilled water, stained with hematoxylin (No. S2020, Dako) for 1 min, washed with water, dehydrated with alcohol (96\%), cleared with xylene, and mechanically covered.

Melanoma and testicular tissues expressing CTAs were used as positive controls. As a negative control, we replaced primary antibodies with isotype matched immunoglobulins. In addition, both antibodies were tested on normal esophageal tissue (resection margins) and lymph node without tumor where showed no positive reaction.

The positive cells were scored in whole tumor at x200 magnification on chosen slides. To evaluate the level of MAGE-A 3/4 and NYESO-1 protein expression, percentages of positive-staining cells and the staining intensity were graded on a scale of $0-3$. Staining percentage was determined as: $0=0 \%$ positive cells; 1 = up to $10 \%$ positive cells; $2 \geq 10$ to $50 \%$ positive cells; and $3 \geq 50 \%$ positive cells. Staining intensity was denoted as: $0=$ no staining; $1=$ weak staining; $2=$ moderate staining; $3=$ strong staining. For each sample, staining percentage and staining intensity scores were multiplied to give the staining index. Immunohistochemical staining index (ISI) was labelled as: $0=$ zero; $1-3=$ low; $4-6=$ moderate and $9=$ high.

All samples were examined independently by three observers and any difference was resolved by a joint review.

\section{Statistical analysis}

Smirnov-Kolmogorov test was used to analyze data distribution prior to statistical analysis. Statistical analysis was performed using $\chi^{2}$-test, Fischer's exact test, Pearson correlation test and Spearman rank correlation coefficients. Statistical significance was determined if the 2-sided value of a test was less than 0.05 .

Statistical analyses were performed using SPSS 15, Statistical Package (SPSS Inc, Chicago, IL, USA).

\section{Results}

Immunohistochemical expression results of primary tumor and lymph node metastasis specimens are summarized in Table 1 and 2. Fifty (90.9\%) primary ESCCs were positive for MAGE-A 3/4 and 53 (96.6\%) were positive for NY-ES0-1. A strong immunohistochemical reaction for MAGE-A 3/4 and NY-ESO-1 was observed in 30 (54.6\%) and 18 (32.7) primary ESCCs, respectively.

MAGE-A 3/4 was expressed in all lymph node metastases and the intensity of expression was high in a majority of cases. NY-ESO-1 was negative in 2 (7.1\%) lymph node metastases, while the reaction was predominantly moderate in the positive group.

The immunohistochemical expression of MAGE-A 3/4 and NY-ESO-1 was restricted to tumor cells and cytoplasmic, both in primary tumors and in lymph node metastases (Figure $1 \mathrm{~A}, \mathrm{~B}, \mathrm{C}, \mathrm{D})$. In primary tumors, MAGE-A $3 / 4$ showed a significantly higher intensity of expression compared to NY-ESO-1 ( $\mathrm{P}=0.047)$, whereas lymph node metastases showed no significant difference in the intensity of expression $(\mathrm{P}=0.387)$. Primary tumors with and without lymph node metastases showed no significant difference in MAGE-A 3/4 $(\mathrm{P}=0.672)$ and NY-ESO-1 $(\mathrm{P}=0.444)$ expression. Intensity of MAGE-A $3 / 4(\mathrm{P}=0.461)$ and NY-ESO-1 $(\mathrm{P}=0.414)$ expression in primary tumors was not significantly different compared to the expression in corresponding

Table 1. Immunohistochemical staining index of MAGE 3/4 and NY-ESO-1 in primary esophageal squamous cell carcinoma.

\begin{tabular}{lcc} 
IST & MAGE 3/4 & NY-ESO-1 \\
0 & $5(9.1 \%)$ & $2(3.6 \%)$ \\
1 & $7(12.7 \%)$ & $19(34.6 \%)$ \\
\hline 2 & $13(23.6 \%)$ & $16(29.1 \%)$ \\
3 & $30(54.6 \%)$ & $18(32.7 \%)$ \\
\hline Total & $55(100 \%)$ & $55(100 \%)$ \\
\hline
\end{tabular}

ISI, immunohistochemical staining index.

Table 2. Immunohistochemical staining index of MAGE 3/4 and NY-ESO-1 in lymph node metastase.

\begin{tabular}{lcc} 
ISI & MAGE 3/4 & NY-ESO-1 \\
0 & $0(0 \%)$ & $2(7.1 \%)$ \\
1 & $8(28.6 \%)$ & $8(28.6 \%)$ \\
\hline 2 & $8(28.6 \%)$ & $13(46.4 \%)$ \\
3 & $12(42.8 \%)$ & $5(17.9 \%)$ \\
\hline Total & $28(100 \%)$ & $28(100 \%)$ \\
\hline
\end{tabular}

ISI, immunohistochemical staining index. 
lymph node metastases.

Expression of MAGE-A 3/4 and NY-ESO-1 showed significant positive correlation within the primary tumor $(\mathrm{P}=0.021)$, but no significant correlation within lymph node metastases $(\mathrm{P}=0.056)$. Also, there was no correlation of MAGE-A 3/4 expression in the primary tumor and lymph node NY-ESO-1 expression $(\mathrm{P}=0.978)$, patient age $(\mathrm{P}=0.904)$, patient sex $(\mathrm{P}=0.499)$, tumor grade $(\mathrm{P}=0.945)$, $\mathrm{T}$ classification $(\mathrm{P}=0.589)$, lymph node status (N classification) $(\mathrm{P}=0.569)$ and distant metastasis $(\mathrm{M}$ classification) $(\mathrm{P}=0.876)$.

Expression of NY-ESO-1 in the primary tumor showed significant positive correlation with NY-ESO-1 lymph node metastasis expression $(\mathrm{P}=0.001)$ and significant negative correlation with patients' age $(\mathrm{P}<0.001)$. Intensity of NY-ESO-1 primary tumor expression corresponded to the intensity of lymph node metastasis expression. Also, the expression of NYESO-1 in primary tumors was much higher in younger patients.

Patient's sex $(\mathrm{P}=0.390)$, tumor grade $(\mathrm{P}=0.962)$, T classification $(\mathrm{P}=0.796), \mathrm{N}$ classification $(\mathrm{P}=0.779)$ and $\mathrm{M}$ classification $(\mathrm{P}=0.437)$ showed no significant correlation with expression of NY-ESO-1 in the primary tumor.

Significant correlation between expression of MAGE-A 3/4 and NY-ESO-1 in the lymph node metastasis was not found $(\mathrm{P}=0.137)$. In addition, the expression of MAGE-A $3 / 4$ and NY-ESO-1 in lymph node metastases did not significantly correlate with patient age $(P>0.05)$, patient sex $(P>0.05)$, tumor grade $(\mathrm{P}>0.05)$, T classification $(\mathrm{P}>0.05), \mathrm{N}$ classification $(\mathrm{P}>0.05)$ or $\mathrm{M}$ classification $(\mathrm{P}>0.05)$.

\section{Discussion}

Several studies have analyzed the expression of MAGE-A and NY-ESO-1 in ESCC. ${ }^{10-14,18-20}$ To our knowledge, this is the first study to assess and compare the expression of MAGE-A and NY-ESO-1 in ESCC lymph node metastases.

MAGE-A was detected in $50-84 \%$ of ESCC, according to different studies. ${ }^{10-14}$

In our study, MAGE-A 3/4 was expressed in 90.9\% primary ESCC and all lymph node metastases. MAGE-A $3 / 4$ was similarly expressed in primary tumor with and without metastases and the intensity of expression was not significantly different between primary tumors and corresponding lymph nodes metastases. Our results are most similar to the results published by Quillien et al., ${ }^{10}$ who assessed the expression of MAGE-A 1,2,3 and 4 genes in 49 ESCC samples by RT-PCR and PCR amplification. MAGE-A 1 was expressed in 53\%, MAGE-A 2 in 49\%, MAGE-A 3 in $47 \%$ and MAGE-A 4 in
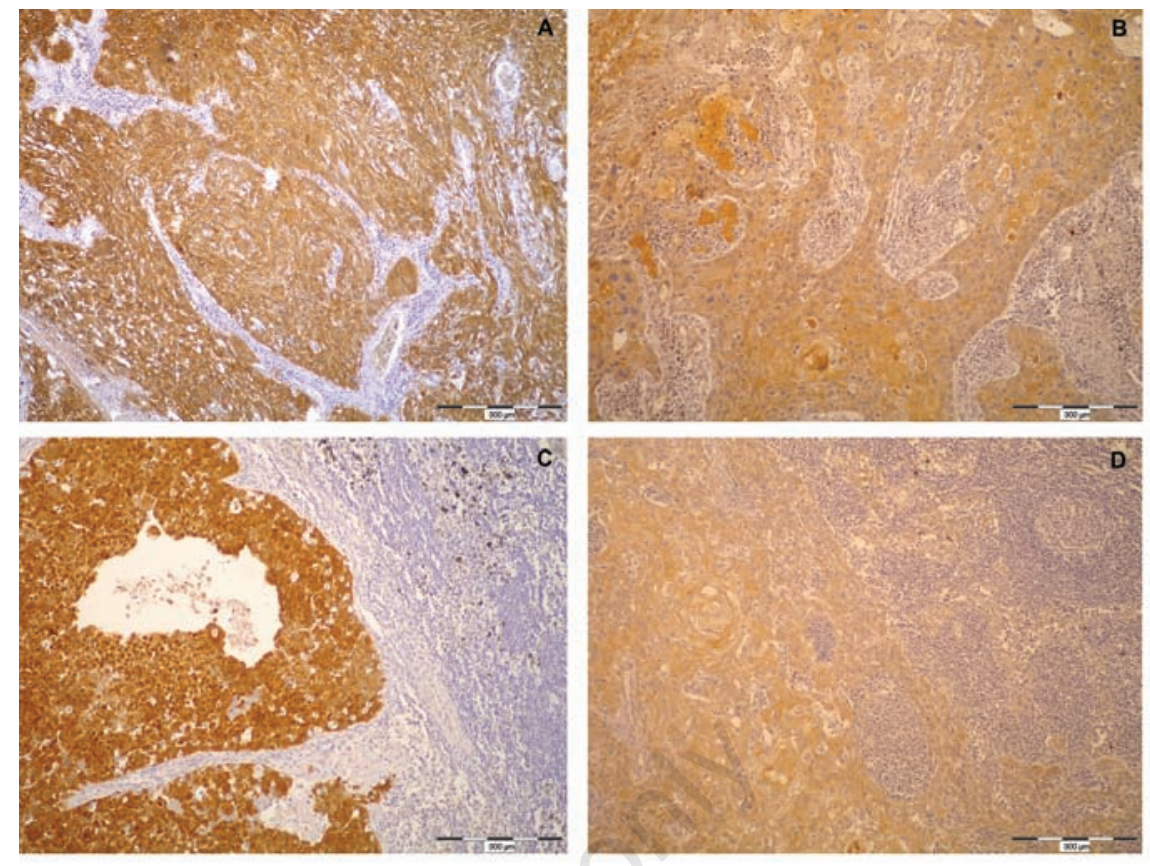

Figure 1. Immunohistochemical expression of MAGE-A 3/4 and NY-ESO-1 in esophageal squamous cell carcinoma and lymph node metastasis. Expression of A) MAGE-A 3/4 and B) NY-ESO-1 in a primary tumor was cytoplasmic and limited to tumor cells. Similar immunohistochemical reaction for C) MAGE-A 3/4 and D) NY-ESO-1 was also observed in corresponding lymph node metastases. Scale bar $=300 \mu \mathrm{m}$.

$71 \%$ tumors, respectively. Eighty four percent of tumors expressed one or more of the investigated MAGE genes. ${ }^{10}$

In another similar study also conducted by RT-PCR, MAGE-A 1, 2 and 3 genes were analyzed in 42 surgical samples and in 12 cell lines of human esophageal carcinoma. ${ }^{11}$ MAGE-A 1, 2 , and 3 genes were expressed in 26 (62\%), 18 (43\%) and 24 (57\%) tumor specimens, respectively. Thirty three of 42 (79\%) carcinomas expressed at least one MAGE gene. In normal esophageal tissue, MAGE-A expression was not found. In cell lines, expression of MAGE-A 1, 2, and 3 was recognized in 5, 4 and 4 cell lines, respectively. ${ }^{11}$

Zambon et al. ${ }^{12}$ analyzed MAGE, BAGE, and GAGE gene expression in 24 ESCC and 24 esophageal adenocarcinomas by RT-PCR and correlated their expression patterns with principal prognostic parameters and long term survival. Sixteen ESCC (67\%) and 9 adenocarcinomas (38\%) expressed at least one of the studied genes. The expression of each MAGE gene in the two histologic types was not significantly different, with the exception of MAGE4, which was expressed more frequently in ESCC samples than in adenocarcinoma samples. BAGE and GAGE expression was low. In each sample it was associated with the expression of at least one MAGE gene. Gene expression was not correlated with disease progression, TNM factors or survival. ${ }^{12}$

Expression of MAGE-A proteins in ESCC was also studied by immunohistochemistry. ${ }^{13,14}$

Immunohistochemical analysis of MAGE-A protein expression in 98 patients with ESCC or adenocarcinoma showed positive reaction in five out of 32 adenocarcinomas (15\%) and in 33 out of 66 (50\%) ESCC. ${ }^{13}$

Similar results of MAGE-A protein expression in esophageal carcinoma was published by Akcakanat et al. ${ }^{14}$ They examined the immunohistochemical expression of CTA proteins in 213 patients with esophageal carcinoma. GAGE, NY-ESO-1 and MAGE-A were heterogeneously expressed in 42 (20\%), 44 (21\%) and 111 (52\%) tumors, respectively, whereas SSX expression was not detected. Expression of MAGE showed correlation with those of GAGE and NY-ESO-1.14

We also found significant positive correlation between MAGE-A 3/4 and NY-ESO-1 expression in primary tumors, and no correlation in lymph node metastases expression. The level of MAGE-A 3/4 expression in primary tumor and corresponding lymph nodes metastases approached the threshold for significant correlation, but did not reach it $(\mathrm{P}=0.056)$.

The lowest expression of NY-ESO-1 in ESCC was reported in the previously mentioned immunohistochemical study conducted by Akcakanat et al. ${ }^{14}$ Similar expression was observed by Mashino et al. who investigated 46 samples of esophageal carcinoma by RT-PCR analysis and found expression in 11 (24\%) esophageal carcinomas. ${ }^{18}$ 
In another larger study which included 123 ESCCs, the expression of NY-ESO-1 mRNA was analyzed by conventional and real-time RTPCR and the expression of protein by immunohistochemistry and Western blot. In addition, sera and peripheral blood lymphocytes from 51 patients were analyzed for the NY-ESO-1 antibody production by enzyme-linked immunosorbent assay and NY-ESO-1 T cell response by enzyme-linked immunospot assay. NY-ESO-1 mRNA was expressed in 41 (33\%) carcinoma specimens and the expression was higher in well-differentiated and moderately differentiated type of carcinoma. Also, twenty-one of 24 (87.5\%) mRNA positive tumors were stained positively by immunohistochemistry. Correlation between the level of NY-ESO-1 mRNA expression and the degree of immunohistochemical positivity was observed. Antibody production was observed in 2 patients with tumors that showed protein expression. Survival data indicated that the survival rate was higher in NY-ES0-1 protein-positive cases than in negative cases, but the difference was not statistically significant. ${ }^{19}$

Akcakanat et al. analyzed the sera of 69 patients with esophageal cancer for antibody production against NY-ESO-1 by Western blot analysis. Moreover, they also analyzed 56 tissue samples for NY-ESO-1 protein expression by immunohistochemistry. NY-ESO-1 protein expression was found in 18 of 56 (32\%) esophageal carcinomas. NY-ESO-1 serum specific immunoreactivity was found in 9 patients (13\%), of whom 8 were in the advanced stage (stages III and IV). They found no relationship between clinico-pathologic features and serum immunoreactivity for NY-ESO-1. NY-ESO-1 protein expression was detected in three of five antibody-positive patients whose tissue was available for analysis but survival analysis showed no significant difference between antibody-positive and antibody-negative patient groups. ${ }^{20}$

In our study, the expression of NY-ESO-1 in primary tumor specimens was higher compared to previously reported results..$^{14,18-20} \mathrm{We}$ found only two tumors and two lymph nodes metastases with a negative reaction for NYESO-1. Currently we are not able to explain this difference in NY-ESO-1 expression but we believe that further investigation could resolve this issue. We found a significant positive correlation between NY-ESO-1 expression in primary tumors and corresponding lymph node metastases. In addition, the expression of NYESO-1 in primary tumors was significantly higher in younger patients. To our knowledge this is a first report of connection between NYES0-1 expression and age in ESCC. Expression of NY-ESO-1 diminishes with age, which would indicate lesser immunogenicity and possible worse response on adjuvant immunotherapy in older patients.

Expression of MAGE-A 3/4 and NY-ESO-1 in ESCC showed no significant correlation with prognostic parameters, such as TNM stage and survival. ${ }^{11,12,14,18-20}$ Only one study found the expression of NY-ESO-1 to be higher in well-differentiated and moderately differentiated ESCC. In this study, the survival rate was higher in NY-ESO-1 positive cases, but did not reach statistical significance. ${ }^{19}$ Our results were similar with previous investigations and the expression of MAGE $3 / 4$ and NYESO-1 in primary tumors and lymph node metastases showed no influence on prognostic parameters.

MAGE-A and NY-ESO-1 expression was also analyzed in prostate carcinoma and squamous cell carcinoma of the penis. ${ }^{16,17}$ In prostate carcinoma multi-MAGE-A and NY-ESO-1 was expressed in $85.9 \%$ and $84.8 \%$ carcinomas while in penile carcinoma $97 \%$ of tumors showed positive reaction on tested antigens but in both carcinomas like in ESCC, expression did not significantly correlate with prognostic parameters. ${ }^{16,17}$

Recent clinical trials have demonstrated a clinical benefit by postoperative vaccine with MAGE-A3 in non-small cell lung cancer and in stage IV melanoma. These trials have led to the current Phase III trials. ${ }^{22,30,31}$

We have shown different levels of MAGE-A $3 / 4$ and NY-ESO-1 expression in almost all specimens of primary tumor and lymph node metastases, suggesting that ESCC may be possible target of immunotherapy and anti-tumor vaccination. High levels of expression in lymph node metastases indicate possible clinical benefit of postoperative vaccine with MAGE-A3 and NY-ESO-1 in advanced stage of disease. Moreover, NY-ESO-1 expression in tumors negatively correlated with patient age which indicating better response on therapy in younger patients.

\section{References}

1. Hamilton SR, Aaltonen LA. WHO classification of tumours. Pathology and genetics of tumours of the digestive system. IARC Press, Lyon, France, 2000.

2. Nozoe T, Saeki H, Ohga T, Sugimachi K. Clinicopathological features of early esophageal squamous cell carcinoma with subsequent recurrence. Dis Esophagus 2002;15:145-8.

3. Scanlan MJ, Gure A0, Jungbluth AA, Old LJ, Chen YT. Cancer/testis antigens: an expanding family of targets for cancer immunotherapy. Immunol Rev 2002;188: 22-3.

4. Zendman AJ, Ruiter DJ, Van Muijen GN.
Cancer/testis-associated genes: identification, expression profile, and putative function. J Cell Physiol 2003;194:272-88.

5. Scanlan MJ, Simpson AJ, Old LJ. The cancer/testis genes: review, standardization, and commentary. Cancer Immun 2004;4:115.

6. Sarcevic B, Spagnoli GC, Terracciano L, Schultz-Thater E, Heberer M, Gamulin M, et al. Expression of cancer/testis tumor associated antigens in cervical squamous cell carcinoma. Oncology 2003;64:443-9.

7. Busam KJ, Iversen K, Berwick M, Spagnoli GC, Old LJ, Jungbluth AA. Immunoreactivity with the anti-MAGE antibody 57B in malignant melanoma: frequency of expression and correlation with prognostic parameters. Mod Pathol 2000;13:45965.

8. Kim SH, Lee S, Lee CH, Lee MK, Kim YD, Shin DH, et al. Expression of cancer-testis antigens MAGE-A3/6 and NY-ESO-1 in nonsmall-cell lung carcinomas and their relationship with immune cell infiltration. Lung 2009;187:401-11.

9. Demirovic A, Dzombeta T, Tomas D, Spajic B, Pavic I, Hudolin T, et al. Immunohistochemical expression of tumor antigens MAGE-A3/4 and NY-ESO-1 in renal oncocytoma and chromophobe renal cell carcinoma. Pathol Res Pract 2010;206:6959.

10. Quillien V, Raoul JL, Heresbach D, Collet B, Toujas L, Brasseur F. Expression of MAGE genes in esophageal squamous-cell carcinoma. Anticancer Res 1997;17:38791.

11. Inoue H, Mori M, Li J, Mimori K, Honda M, Nakashima H, et al. Human esophageal carcinomas frequently express the tumorrejection antigens of MAGE genes. Int $\mathrm{J}$ Cancer 1995;63:523-6.

12. Zambon A, Mandruzzato S, Parenti A, Macino B, Dalerba P, Ruol A, et al. MAGE, BAGE, and GAGE gene expression in patients with esophageal squamous cell carcinoma and adenocarcinoma of the gastric cardia. Cancer 2001;91:1882-8.

13. Haier J, Owzcareck M, Guller U, Spagnoli GC, Bürger H, Senninger N, et al. Expression of MAGE-A cancer/testis antigens in esophageal squamous cell carcinomas. Anticancer Res 2006;26:2281-7.

14. Akcakanat A, Kanda T, Tanabe T, Komukai S, Yajima K, Nakagawa S, et al. Heterogeneous expression of GAGE, NY-ESO-1, MAGE-A and SSX proteins in esophageal cancer: Implications for immunotherapy. Int J Cancer 2006;118: 123-8.

15. Zeng G, Aldridge ME, Wang Y, Pantuck AJ, Wang AY, Liu YX, et al. Dominant B cell epitope from NY-ESO-1 recognized by sera from a wide spectrum of cancer patients: 
implications as a potential biomarker. Int $\mathrm{J}$ Cancer 2005;114:268-73.

16. Hudolin T, Juretic A, Spagnoli GC, Pasini J, Bandic D, Heberer M, et al. Immunohi stochemical expression of tumor antigens MAGE-A1, MAGE-A3/4, and NY-ESO-1 in cancerous and benign prostatic tissue. Prostate 2006;66:13-8.

17. Hudolin T, Juretic A, Pasini J, Tomas D, Spagnoli GC, Heberer M, et al. Immunohistochemical expression of tumor antigens MAGE-A1, MAGE-A3/4, and NY-ESO-1 in squamous cell carcinoma of the penis. Urology 2006;68:205-7.

18. Mashino K, Sadanaga N, Tanaka F, Yamaguchi $\mathrm{H}$, Nagashima $\mathrm{H}$, Inoue $\mathrm{H}$, et al. Expression of multiple cancer-testis antigen genes in gastrointestinal and breast carcinomas. Br J Cancer 2001;85: 713-20.

19. Fujita S, Wada H, Jungbluth AA, Sato S, Nakata T, Noguchi Y, et al. NY-ESO-1 expression and immunogenicity in esophageal cancer. Clin Cancer Res 2004;10:6551-8.

20. Akcakanat A, Kanda T, Koyama Y, Watanabe M, Kimura E, Yoshida Y, et al. NY-ESO-1 expression and its serum immunoreactivity in esophageal cancer. Cancer Chemother Pharmacol. 2004;54:95100.

21. Caballero OL, Chen YT. Cancer/testis (CT) antigens: potential targets for immunotherapy. Cancer Sci. 2009;100: 2014-21.

22. Peled N, Oton AB, Hirsch FR, Bunn P. MAGE A3 antigen-specific cancer immunotherapeutic. Immunotherapy 2009;1:1925.

23. Cebon J, Knights A, Ebert L, Jackson H, Chen W. Evaluation of cellular immune responses in cancer vaccine recipients: lessons from NY-ESO-1. Expert Rev Vaccines 2010;9:617-29.

24. West HJ. Novel targeted agents for lung cancer. Clin Lung Cancer. 2009;10:S41-6.

25. Sobin LH, Wittekind CH. TNM classification of malignant tumors. 6th ed. WileyLiss, New York, USA, 2002.

26. Kocher T, Schultz-Thater E, Gudat F, Schaefer C, Casorati G, Juretic A, et al. Identification and intracellular location of MAGE-3 gene product. Cancer Res 1995; 55:2236-9.

27. Juretic A, Spagnoli GC, Schultz-Thater E, Sarcevic B. Cancer/testis tumourassociated antigens: immunohistochemical detec- tion with monoclonal antibodies. Lancet Oncol 2003;4:104-9.

28. Landry C, Brasseur F, Spagnioli GC, Marbaix E, Boon T, Coulie $\mathrm{P}$ et al. Monoclonal antibody 57B stains tumor tissues that express gene MAGE-A4. Int $\mathrm{J}$ Cancer 2000;86:835-41.

29. Schultz-Thater E, Noppen C, Gudat F, Dürmüller U, Zajac P, Kocher T, et al. NYESO-1 tumour associated antigen is a cytoplasmic protein detectable by specific monoclonal antibodies in cell lines and clinical specimens. Br J Cancer 2000;83: 204-8.

30. Gridelli C, Rossi A, Maione P, Ferrara ML, Castaldo V, Sacco PC. Vaccines for the treatment of non-small cell lung cancer: a renewed anticancer strategy. Oncologist 2009:14:909-20.

31. Tyagi P, Mirakhur B. MAGRIT: the largestever phase III lung cancer trial aims to establish a novel tumor-specific approach to therapy. Clin Lung Cancer 2009;10:371-4 